\title{
Concurrent validity of nutrition risk scores in paediatric inflammatory bowel disease
}

\author{
D. R. Owens ${ }^{1}$, A. E. Wiskin ${ }^{1}$, V. R. Cornelius ${ }^{1}$, S. A. Wootton ${ }^{1}$ and R. M. Beattie ${ }^{2}$ \\ ${ }^{1}$ NIHR Nutrition, Diet and Lifestyle Biomedical Research Unit, Southampton SO 17 6YD, UK and \\ ${ }^{2}$ Paediatric Medical Unit, Southampton SO17 6YD, UK
}

Nutritional care is a priority in the management of children with inflammatory bowel disease (IBD) in whom deficits in weight and linear growth are common. Several paediatric nutrition risk scores have recently been published. These tools categorise children into low-, moderate- and high-nutritional risk. Although these scores share many components, the level of agreement between scores in children with IBD is uncertain.

This study examined the concurrent validity of nutrition screening tools within the IBD clinic. Four nutritional screening tools: STAMP $^{(1)}$, STRONGkids ${ }^{(2)}$, PYMS ${ }^{(3)}$, Simple Pediatric Nutritional Risk Score (SPNRS) ${ }^{(4)}$ were consolidated into one generic assessment from which the scores for each tool were derived. Children with IBD, confirmed by histopathological criteria, attending the regional paediatric gastroenterology service were studied with this generic assessment by a single trained observer. Nutritional risk was determined from each tool. Kappa statistics were used to find the level of agreement between each risk score compared to that expected by chance.

Forty-six children were studied, 24 boys. Median age was 14 years (range 3-17 years). Twenty-seven children had Crohn's, 16 UC and 3 Indeterminate Colitis. PYMS was the only tool to place children at low risk, 23 in total; 1 scored low with PYMS and high with STAMP, 4 scored low with PYMS and high with SPNRS.

\begin{tabular}{lccc}
\hline STRONGkids & SPNRS & PYMS & \\
\hline STAMP & 0.774 & 0.732 & 0.332 \\
STRONGkids & & 0.6 & 0.270 \\
SPNRS & & & 0.236 \\
\hline
\end{tabular}

Kappa scores to show the level of agreement of nutrition risk scores in 46 children with IBD. A kappa score $>0.6$ represents a good level of agreement, $<0.2$ is poor.

There was good agreement between STAMP, STRONGkids and SPRNS; however, no children received a low-risk score with these tools. Children with IBD were automatically scored medium risk, due to their disease, with these three tools. PYMS scored most children at low risk and therefore did not have a good level of agreement with the other scores. The potential clinical application of any of these tools requires further study.

1. http://www.stampscreeningtool.org/stamp.html

2. Hulst JM, Zwart H, Hop WC et al. (2010) Clin Nutr 29, 106-111.

3. Gerasimidis K, Keane O, Macleod I et al. (2010) Br J Nutr 19, 1-6.

4. Sermet-Gaudelus I, Poisson-Salomon AS, Colomb V et al. (2000) Am J Clin Nutr 72, 64-70. 